\title{
Arm im Darm
}

\section{Microbiom, Mangelernährung und mehr ${ }^{1}$}

\section{Spitzer, Ulm}

Von Mangelernährung bei Armut und den dadurch bedingten massiven Beeinträchtigungen der Entwicklung von Körper, Gehirn und Geist war an dieser Stelle bereits mehrfach die Rede $(53,54)$. Dabei wurde klar: Unterernährung schadet Kindern deswegen mehr als Erwachsenen, weil sie deren Entwicklung zum normalen Erwachsenen beeinträchtigt und daher teilweise irreparable Schäden nach sich zieht.

Aus meiner Kindheit sind mir noch die vielen schrecklichen Bilder in Nachrichten über den damals in Nigeria tobenden Bürgerkrieg mit der abtrünnigen Provinz Biafra (Biafra-Krieg vom 6. Juli 1967 bis 15. Januar 1970) in Erinnerung, in denen immer wieder völlig abgemagerte kleine Kinder mit dicken Bäuchen zu sehen waren $(\triangleright A b b .1)$. Erst im Studium lernte ich, dass sie an einer rätselhaften Krankheit namens Kwashior$k r^{2}$ litten, bei der die Leber aus Protein-

1 Den Anstoß zu den Recherchen für diese kleine Übersicht gab ein Vortrag (Presidential Lecture) des an der Washington University in St. Louis arbeitenden US-amerikanischen Mikrobiologen Jeffrey Ivan Gordon $\left({ }^{\star} 1947\right)$ auf dem Kongress der Society for Neuroscience in Washington, DC, am 13.11.2017 (21). Herr Gordon arbeitet seit über 20 Jahren zum Mikrobiom von Tier und Mensch. Studien aus seiner Arbeitsgruppe leisteten einen wesentlichen Beitrag zur Aufklärung der Bedeutung der Darmflora für Gesundheit und Krankheit. Mich würde nicht wundern, wenn er irgendwann den Nobelpreis für Medizin bekäme.

2 Der Name der Krankheit stammt von der Erstbeschreiberin dieser Krankheit, der jamaikanischen Kinderärztin Cicely D. Williams, die in einer im Fachblatt Lancet im Jahr 1935 publizierten Arbeit auch gleich die Bedeutung des Wortes klärt: Das Wort bedeutet in der an der Goldküste (wie die britische Kolonie auf dem Gebiet des heutigen Ghana damals genannt wurde) gesprochenen Sprache soviel wie „die Krankheit, die ein Kind bekommt, wenn ein neues Kind geboren wird“ (67, S. 1151).

Nervenheilkunde 2018; 37: 73-80

Korrespondenzadresse

Prof. Dr. Dr. Manfred Spitzer, Universitätsklinikum Ulm Klinik für Psychiatrie und Psychotherapie III

Leimgrubenweg 12, $89075 \mathrm{Ulm}$ mangel das aufgenommene Fett nicht abgeben kann und daher in sich anreichert. Zugleich bestehen Eiweißmangelödeme und Anämie, eine Entfärbung der Haare und eine dünne Haut sowie eine generelle Apathie - einschließlich Appetitmangel.

Heute spricht man von schwerer akuter Unterernährung - severe acute malnutrition (SAM) - oder von mäßiger akuter Unterernährung - moderate acute malnutrition (MAM). Beide Störungsbilder wurden von der Weltgesundheitsorganisation (WHO) statistisch als Abweichung vom normalen

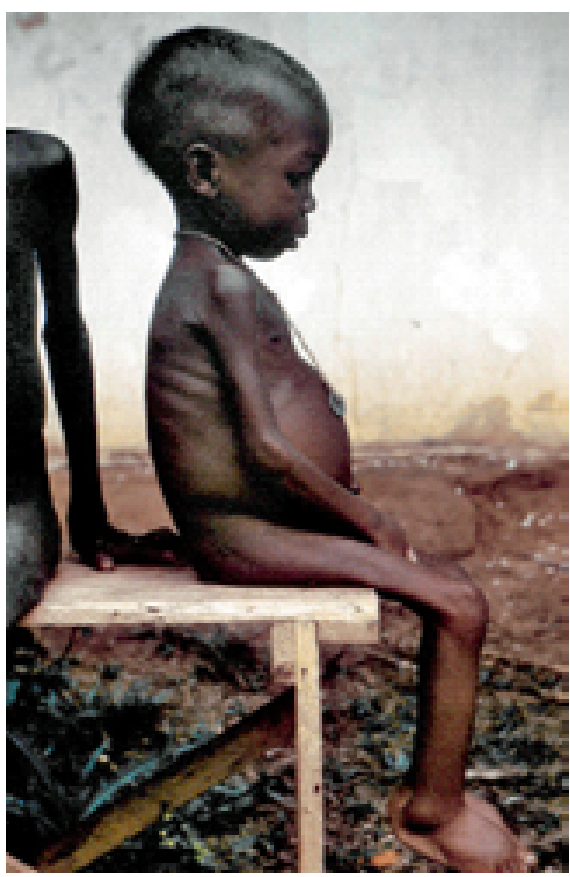

Abb. 1 Kleines Mädchen im Biafra-Krieg, das infolge von Unterernährung an Kwashiorkor leidet. Das Bild wurde Ende der 1960er-Jahre von Dr. Lyle Conrad vom Center for Disease Control and Prevention (Atlanta, Georgia, USA) aufgenommen und ist Teil der Public Health Image Library (PHIL) (ID: 6901 http://phil.cdc.gov/). [Einzelheiten zur Genehmigung: This image is in the public domain and thus free of any copyright restrictions. As a matter of courtesy we [the Centers for Disease Control and Prevention] request that the content provider be credited and notified in any public or private usage of this image.]
Verhältnis von Körpergewicht zu Körpergrö$\mathrm{Be}^{3}$ definiert. Die Unterernährung tritt in der Regel im Alter von drei Monaten bis zwei Jahren auf und hat beim Vorliegen der schweren Form (SAM) eine hohe Mortalität von 20-50\% innerhalb von zwei Tagen. Daher wurde schon vor zwei Jahrzehnten eine standardisierte Behandlung (mit einer hochkalorischen Diät aus Erdnusspaste, Zucker, Pflanzenöl und mit Vitaminen und Mineralien angereicherter Milch) eingeführt, mit der sich die Sterblichkeit auf weniger als $10 \%$ verringern ließ, wie empirische Studien zeigten (1).

\section{Von Mangelernährung betroffene Kleinkinder reagieren keineswegs immer auf die Verbesserung ihrer Ernährung mit einer Gewichtszu- nahme.}

Von Mangelernährung betroffene Kleinkinder reagieren jedoch keineswegs immer auf die Verbesserung ihrer Ernährung mit einer - eigentlich $\mathrm{zu}$ erwartenden - Gewichtszunahme. Dies führte dazu, dass Kwashiorkor lange als „rätselhafte Krankheit" bezeichnet wurde. Als dann auch noch eine vor zehn Jahren publizierte, in Malawi durchgeführte, sehr sorgfältige ${ }^{4}$

3 Sowohl Körpergewicht als auch Körpergröße werden hierzu zunächst z-transformiert, d. h. auf einen Mittelwert von 0 und eine Standardabweichung von 1 gebracht. Man spricht hier auch vom weight-forheight Z-score (WHZ). Liegt dieser Wert mehr als drei Standardabweichungen unter dem Median, spricht man von SAM, liegt er zwischen zwei und drei Standardabweichungen unter dem Median, spricht man von MAM.

4 Zum Beleg sei aus der Zusammenfassung zitiert: „Dietary intake was assessed on enrollment using a food frequency questionnaire, which included all possible foods in the child's diet. Food frequency data were used to estimate energy, protein, vitamins $\mathrm{C}$ and $\mathrm{A}$, niacin, thiamin, zinc, and iron intake using food composition and serving size data. [...]In the combined regression model no foods or nutrients were found to be associated with the development of kwashiorkor. There were no differences in the dietary diversity between children who developed kwashiorkor and those who did $\operatorname{not}^{\text {“ }}(32$, S. 487). 
Längsschnittstudie an 1651 gesunden Kindern im Alter von ein bis drei Jahren ergab, dass es keinen Zusammenhang zwischen der Ernährung der Kinder und der Entwicklung von Kwashiorkor gibt, war das Rätsel auf den Punkt gebracht: Die Krankheit besteht in einer „Mangelernährung“ ohne dass ein Mangel in der Ernährung vorliegen muss. Wie kann das sein?

\section{Szenenwechsel}

Ein Mensch besteht aus etwa $3 \times 10^{13}, \mathrm{~d}$. h. 30000 Milliarden Zellen. In seinem Dickdarm leben etwa $3,8 \times 10^{13}$ Bakterien - also 38000 Milliarden $(49)^{5}$. Bis vor wenigen Jahren sprach man bescheiden von der Darmflora und meinte damit das, was in der Petrischale (unter verschiedenen, meist aeroben Bedingungen) wächst, wenn man sie mit einer Stuhlprobe beimpft: E. coli dürfte unser bekanntester Bewohner sein.

Nachdem die Fortschritte der modernen Genetik zur Identifizierung des menschlichen Genoms geführt hatten (13, $19,63)$ und fast täglich die Genome weiterer Organismen erfasst und katalogisiert wurden, kam die Idee auf, auch die Gesamtheit unserer Bewohner mit genetischen Methoden zu untersuchen. Der Name für das untersuchte „Objekt", unser Microbiom, geht auf den US-Amerikaner Joshua Lederberg (1925-2008), einem Mikrobiologen und Genetiker (1958 Nobelpreis für Medizin), zurück - analog zum unserem Genom. Die Initiativen zur genaueren Beschreibung der auf und in uns lebenden Bakterien ${ }^{6}$ durch genetische Methoden resultierten schließlich im Human Microbiome Project, das 2008 vom USamerikanischen National Institute of Health (NIH) mit einem Gesamtbudget von 115 Millionen US Dollar gestartet wurde (73).

Bereits nach vier Jahren waren etwa 5000 Proben von rund 240 Erwachsenen

5 Nichts ist in Stein gemeißelt: Zehn Jahre vor der Publikation (und sehr weiten Verbreitung) dieser Zahlen wurde das Verhältnis von menschlichen Zellen $\left(10^{13}\right)$ zu Darmbakterien $\left(10^{14}\right)$ auf „etwa 1:10" geschätzt. Seither geistert die Auffassung, wir hätten zehnmal mehr Bakterien im Darm als Zellen im gesamten Körper, durch die Literatur. untersucht (von jedem wurden etwa 20 Proben genommen) und die beteiligten Wissenschaftler erlebten eine Überraschung $(57,58)$ : Auf und in jedem von uns leben etwa 10000 unterschiedliche Arten von Bakterien, und damit sehr viel mehr Arten als man zuvor angenommen hatte. Der Genpool des menschlichen Microbioms ist mit 8 Millionen Genen (d. h. für Proteine kodierende DNA-Sequenzen) 360-mal größer (!) als das menschliche Erbgut mit seinen gerade mal etwa 22000 Genen. Rein biochemisch betrachten „können" die Gene unserer Bewohner zusammen genommen damit wesentlich mehr als wir.

\section{Der Genpool des menschlichen Microbioms ist mit 8 Millionen Genen 360-mal größer als das menschliche Erbgut.}

So wundert es nicht, dass unsere Darmflora einen Teil des Stoffwechsels für uns erledigt, d. h. teilweise die Biochemie übernimmt, die sonst in unserer Leber oder in anderen Zellen unseres Körpers ablaufen müsste, aber nicht ablaufen kann, weil uns Menschen die Enzyme dafür fehlen. Unsere Darmbakterien produzieren sogar manche Vitamine $\left(\mathrm{B}_{1}, \mathrm{~B}_{2}, \mathrm{~B}_{6}, \mathrm{~B}_{12}\right.$ und $\left.\mathrm{K}\right)$, also Stoffe, die wir definitionsgemäß gar nicht selbst produzieren können. Darmbakterien bauen für uns unverdauliche Ballaststoffe ab, u. a. zu kurzkettigen Fettsäuren wie Essigsäure, Buttersäure und Propionsäure (Acetat, Butyrat, Propionat), was bis zu 10\% un-

6 Schon lange hatten Mikrobiologen mittels Bakterienkulturen im Labor Bakterien identifiziert, die nicht nur im, sondern auch auf dem Körper des Menschen leben. Um all diese Bakterien (z. B. in der Mundhöhle, unter den Achseln, unter den Nägeln, auf Haut und den Haaren) ging es beim $\mathrm{Hu}$ man Microbiome, wobei die Darmbakterien zahlenmäßig und wahrscheinlich auch im Hinblick auf ihre Bedeutung für unsere Gesundheit den Löwenanteil ausmachen. Streng genommen kann man die Gesamtheit der Bakterien („Mikrobiota“) und die Gesamtheit von deren Genen („Microbiom“) unterscheiden, da der Fortschritt jedoch gerade in der Nutzung genetischer (und nicht mikrobiologischer) Methoden zur Identifikation bestand, wird zumindest im deutschen Sprachraum das Wort „Microbiom“ sowohl für die Gesamtheit der Organismen als auch für die Gesamtheit von deren Genen („Genom") verwendet. serer täglichen Kalorienzufuhr ausmachen kann $(8,36)$. Dies erklärt, dass sich Menschen in ihrer Eigenschaft, bessere oder schlechtere „Kostverwerter" ${ }^{\text {"7 }}$ zu sein, unterscheiden. Insbesondere die Buttersäure und Propionsäure werden beim Abbau von Ballaststoffen (vor allem im aufsteigenden Kolon) gebildet. Sie tragen übrigens auch zum Sättigungsgefühl bei ${ }^{8}$, fördern die Insulinsekretion und hemmen zugleich die Glukagonbildung in der Bauchspeicheldrüse. Unser Microbiom kann also einen antidiabetischen Effekt haben. Auch die Verträglichkeit einer fettreichen Diät hängt von ihm ab und die Insulinresistenz ebenfalls.

Darüber hinaus können Darmbakterien Fremdstoffe entgiften, das Immunsystem stimulieren, Entzündungen bekämpfen und Krankheitserreger entweder einfach zahlenmäßig verdrängen oder sogar aktiv bekämpfen. Ein Beispiel: Rezidivierende Darminfektionen mit Clostridium difficile wurden früher antibiotisch mit Vancomycin behandelt (Erfolgsquote von 30\%), wohingegen die Therapie heute im Einbringen normaler Darmbakterien in den infizierten Darm besteht, die in 85\% der Fälle zum Erfolg führt (34).

Neben dem menschlichen Microbiom wurden mittlerweile u. a. auch noch die Microbiome der Maus, Kuh, Biene und des Schweins beschrieben. Sie unterscheiden sich und sind für ein Verständnis des Stoffwechsels der jeweiligen Art wichtig geworden.

\footnotetext{
7 Diesen Terminus technicus verdanke ich meiner Großmutter, die ohne jegliche Kenntnis des Microbioms ganz selbstverständlich von guten und schlechten Kostverwertern sprach. Nachgewiesen ist jedoch auch die andere Richtung der Kausalität: Das Microbiom unseres Darms reagiert auf unterschiedliche Diäten, teilweise innerhalb von 24 Stunden, wie im Jahr 2011 in Science publiziert wurde (69).

8 Sowohl im Tiermodell (41) als auch beim Menschen (12) konnte gezeigt werden, dass Ballaststoffe über ihren Abbau zu Propionsäure und die dadurch hervorgerufenen Auswirkungen auf den Blutzuckerspiegel und die Sättigung gewichtsreduzierend wirken. Das ist letztlich der Grund, warum Ballaststoffe beim Abnehmen eine so wichtige Rolle spielen.
} 
Das Microbiom des Darms ist verglichen mit der Haut oder anderen Schleimhäuten in höchstem Ausmaß „individualisiert".

Aber auch die Mikrobiome einzelner Individuen unterscheiden sich - ebenso wie deren Genome -, wobei das Mikrobiom des Darms (verglichen mit der Haut oder anderen Schleimhäuten) in höchstem Aus$\mathrm{maß}$,individualisiert“ ist (33). Die Gründe für diese Unterschiede sind beim Menschen vielfältig und reichen von Unterschieden in der frühen Besiedlung gleich nach der Geburt (durch Lactobacillus von der Scheide der Mutter bei normaler Geburt; nach Kaiserschnitt findet dagegen eine Besiedlung mit fremden Keinem statt) über Unterschiede der Bakterien in der Umgebung, vor allem im Boden (in Städten anders als auf dem Land), bis zu den Ernährungs- und Lebensgewohnheiten einschließlich eingenommener Medikamente $^{9}(28,56,59)$.

Bei aller interindividuellen Verschiedenheit der Darmflora konnte mittlerweile jedoch auch gezeigt werden, dass sie gewissen allgemeinen Gesetzmäßigkeiten unterliegt. So hat das Microbiom eine selbststabilisierende Funktion, d. h. es unterliegt bestimmten Fließgleichgewichten, die jeweils stabil sind, sich jedoch durch bestimmte diätetische Maßnahmen (Präbiotika) oder durch das Einbringen anderer Bakterien in den Darm ändern lassen. Weil solche Fließgleichgewichte stabil sind, können auch krankhafte Veränderungen sich selbst stabilisieren. Andererseits können starke Manipulationen des Microbioms (z. B. nach Microbiom-Transplantationen) langfristige Auswirkungen haben, weil sich dadurch ein neues Fließgleichgewicht eingestellt hat. Man konnte dies mittlerweile durch Computersimulationen der Dynamik von Bakteriengemeinschaften nachweisen (7).

9 Hier sind Antibiotika zu nennen, durch die nicht nur resistente Stämme in Nutztieren und beim Menschen gezüchtet werden. Auch abgesehen von gezüchteten Resistenzen beeinflussen Antibiotika die Zusammensetzung unserer Darmflora massiv. Der Einsatz von Protonenpumpenhemmern beim Menschen führt übrigens ebenfalls zu Veränderungen des Microbioms, die krankheitsbegünstigend wirken können (37).
Die Folgen von Unterschieden in der Besiedelung des Darms wurden in den vergangenen Jahren immer genauer untersucht: „Beimpft" man den Darm steril aufgezogener Mäuse mit den Darmbakterien gesunder Mäuse, so nehmen sie ohne vermehrte Nahrungsaufnahme zu $(44,50)$. Darmbakterien bestimmen also tatsächlich darüber mit, ob Mäuse - oder auch Menschen (27) - übergewichtig oder normalgewichtig sind.

Darmbakterien bestimmen tatsächlich darüber mit, ob Mäuse - oder auch Menschen - übergewichtig oder normalgewichtig sind.

Zurück zur Mangelernährung. Eine im Jahr 2012 im Fachblatt Nature publizierte Studie an 531 gesunden Kindern und Erwachsenen aus den USA, Venezuela und Malawi lieferte erste deutliche Hinweise auf die Bedeutung des menschlichen Microbioms für die Entstehung von Mangelernährung. Da von der gleichen Arbeitsgruppe um den US-Mikrobiologen Jeffrey I. Gordon schon sechs Jahre zuvor gefunden worden war, dass das Microbiom auch mit Übergewicht assoziiert war, hatte dieser eine Langzeitstudie an 317 Zwillingspaaren aus Malawi, einem der afrikanischen Länder mit der höchsten Kindersterblichkeit, durchgeführt (51). Von den insgesamt 634 Kindern, die in einem Alter deutlich unter drei Jahren in die Studie aufgenommen wurden und dann bis zum Erreichen des vierten Lebensjahres nachbeobachtet wurden, entwickelten 7,4\% Kwashiorkor und weitere 13,9\% eine mäßige akute Unterernährung (MAM). Man untersuchte die Darmflora von 9 gleichgeschlechtlichen gesunden Zwillingspaaren (5 eineiig, 4 zweieiig; Alter zu Beginn zwischen 3 Wochen und 27 Monaten) und verglich sie mit der Darmflora von 13 Zwillingspaaren (7 eineiig, 6 zweieiig; Alter zu Beginn zwischen 3 Wochen und 30 Monaten), von denen jeweils einer an Kwashiorkor erkrankt war, zu mehreren Zeitpunkten. Hierbei zeigte sich bei den gesunden Kindern eine Entwicklung des Darm-Microbioms in Richtung zunehmender „Reifung ${ }^{\text {“10 }}$ im Hinblick auf die Zusammensetzung, die bei erkrankten Kindern nicht zu beobachten war. Irgendeine Veränderung der Darmflo- ra schien also mit der Mangelernährung in Verbindung zu stehen, aber es war nicht einfach die „Infektion“ mit diesem oder jenem Bakterium. Dieses hätte man längst gefunden, wenn die Sache so einfach gewesen wäre. Vielmehr ging es um eine Veränderung der Zusammensetzung des Microbioms, das sich, wie bereits erwähnt, aus Tausenden unterschiedlicher Bakterien zusammensetzt.

Um die Wirkung des Microbioms auf die Mangelernährung dennoch ursächlich (wenn man so will: „ein für alle Mal“) nachzuweisen, gingen die Autoren wie folgt vor: Sie verwendeten gnotobiotische ${ }^{11}$, also seit der Geburt keimfrei aufgezogene, Mäuse als Krankheitsmodel für Mangelernährung. Mittels einer ziemlich komplizierten Prozedur erhielten die keimfreien Mäuse eine kleine Menge der (zuvor aufbereiteten) tiefgefrorenen Stuhlproben von einem von jeweils drei Kindern, die entweder gesund waren oder an Kwashiorkor litten. Bereits eine Woche zuvor wurden alle Tiere mit einer typischen Diät, wie sie Kinder in Malawi zu sich nehmen, gefüttert, natürlich in sterilisierter Form. Bei den Mäusen, denen Bakterien von Kindern mit Kwashiorkor verabreicht worden waren, kam es, verglichen mit den Mäusen, die Bakterien von gesunden Kindern bekommen hatten, zu einer signifikanten Gewichtsabnahme in den nachfolgenden knapp drei Wochen ( $\triangleright$ Abb. 2). Dann erhielten alle Tiere für zwei Wochen die beschriebene kalorienreiche Diät. Sie nahmen dadurch wieder an Gewicht zu, die mit den Darmbakterien der Kinder mit Kwashiorkor jedoch weniger als die Mäuse

10 Dies wurde mit einem statistisch definierten $\mathrm{Maß}$ gemessen, auf dessen Einzelheiten hier nicht eingegangen werden kann. Auch weitere Einzelheiten des Vorgehens sind methodisch recht komplex und erschließen sich nur nach gründlicher Lektüre auch der Supplementary Materials.

11 Die Gnotobiotik ist die Wissenschaft von keimfrei aufgezogenen Versuchstieren. Das Wort hat zwei griechische Wurzeln - gnōsis: wissen, kennen; und bios: Leben. Weil Tiere wie auch Menschen zwar steril zur Welt kommen, jedoch ab dem ersten Augenblick danach mit Keimen besiedelt werden, die man nicht kennt, haben steril geborene und aufgezogene Versuchstiere diese Bezeichnung: Weil nichts hinzugekommen ist, das man nicht kennt, kennt man deren Microbiom. 


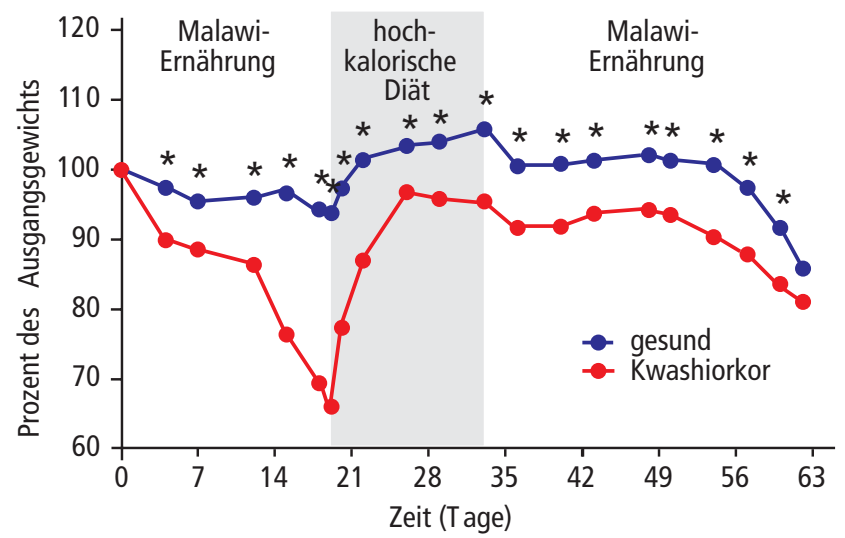

Abb. 2 Verlauf des Körpergewichts von Mäusen, die zunächst steril aufwuchsen und dann (am $\operatorname{Tag} 0$ ) Darmbakterien von Kindern intragastrisch verabreicht bekamen, die entweder mangelernährt waren (rot) oder gesund (blau). Wie die rote Kurve deutlich zeigt, nahmen Mäuse ab, wenn man die Darmbakterien von mangelernährten Kindern in ihren Darm einbringt. Selbst nach einer zweiwöchigen hochkalorischen Diät ist die Gewichtsabnahme nicht voll ausgeglichen und stellt sich nach Umstellung auf die gewöhnliche Kost wieder stärker ein. (Jede Gruppe bestand aus 10 Tieren; nach 52, S. 551, Figure 2A).

(jeweils 10 pro Gruppe) mit den Darmbakterien der gesunden Kinder.

Differenzierte Analysen des Kohlehydrat-, Protein- (essenzielle und nicht essenzielle Aminosäuren), Fett- und NukleotidStoffwechsels der Tiere ergaben Microbiom-bedingte Unterschiede, die erklären konnten, warum ein Kind trotz kalorisch ausreichender Ernährung nicht gedeiht. Damit war die kausale Rolle des Microbioms bei der Entstehung von Mangelernährung im Tiermodell nachgewiesen.

Die Entwicklung bzw. Reifung der menschlichen Darmflora und deren Störungen wurden damit zu einem wichtigen Forschungsgebiet. Die Arbeitsgruppe um Jeffrey Gordon publizierte im Jahr 2014 im Fachblatt Nature hierzu eine Studie, in deren Rahmen bei 50 gesunden Kindern aus Bangladesch (darunter 11 Zwillingspaare und einmal Drillinge) ab dem Zeitpunkt der Geburt für zwei Jahre monatlich Stuhlproben genommen wurden. Hierdurch wurde zunächst eine "gesunde Entwicklung" der Darmflora charakterisiert (71). Man entwickelte hierzu ein Maß - der „,microbiota-for-age Z-score" - für das mikrobiotische Alter eines Kindes, das zum tatsächlichen Alter in Beziehung gesetzt werden kann. ${ }^{12}$

Wie das afrikanische Land Malawi gehört das südasiatische Bangladesch zu den von Hunger weltweit am stärksten betroffenen Regionen. Daher wurden 64 schwer akut mangelernährte Kinder im Alter von 6 bis 20 Monaten vor, während und nach zwei unterschiedlichen Therapie-Diäten (die randomisiert eingesetzt wurden) im Hinblick auf den Entwicklungszustand ihres Microbioms untersucht. Hierbei zeigte sich ein Rückstand in der Entwicklung ihres Microbioms, der sich unter beiden Therapien zwar verringerte, danach jedoch wieder zunahm ( Abb. 3). Beide Therapien führten auch zu einer Gewichtszunahme, die Kinder blieben jedoch deutlich untergewichtig. Der Entwicklungszustand des Microbioms wurde damit als wesentlicher Faktor der Wirksamkeit einer Diät gegen Unterernährung identifiziert. Der Vergleich von Daten aus Bangladesch mit Daten aus Malawi zeigte weiterhin eine große Übereinstimmung der dysfunktionalen Microbiota aus diesen vollkommen unterschiedlichen Regionen der Welt, was die Allgemeinheit der Ergebnisse unterstreicht.

12 Letztlich hat man sich hier an der Intelligenzforschung angelehnt, wo man ja auch das Intelligenzalter zum tatsächlichen Lebensalter in Beziehung setzt, um den Entwicklungszustand der Intelligenz eines Kindes zu beurteilen. Der Intelligenzquotient ist das Verhältnis aus Intelligenzalter und tatsächlichem Lebensalter.
Derzeit laufende Studien aus der gleichen Arbeitsgruppe gehen der Frage nach, ob bestimmte in der lokalen Diät (in Malawi oder Bangladesch) vorhandene Nährstoffe einen günstigen (oder ungünstigen) Effekt auf das Microbiom unterernährter Kinder haben können (21). Um dies herauszufinden ohne schwerkranken Kindern zu schaden, werden nicht diese mit den verschiedensten probiotischen Nahrungsbestandteilen bzw. Kombinationen davon behandelt, sondern deren Stuhlproben auf keimfreie Mäuse übertragen und diese dann den unterschiedlichsten „Behandlungen" mit Kombinationen präbiotischer Nahrungsergänzungsstoffe ausgesetzt. Deren Wirksamkeit lässt sich so im Tierversuch leichter feststellen - man hat schließlich alle Parameter der Diät und der Darmflora unter strenger Laborkontrolle.

Ziel ist es dabei explizit nicht, durch diese Forschung einigen großen Lebensmittelkonzernen zu noch größeren Gewinnen zu verhelfen, sondern dazu beizutragen, lokal produzierte Nahrungsmittel bzw. Prä- oder Probiotika $^{13}$ effektiver einzusetzen. Nur dann finden solche Projekte auch die Unterstützung der lokalen Bevölkerung, die man wiederum zu einem späteren Zeitpunkt für die Übertragung der Erkenntnisse aus diesen Tierversuchen auf den Menschen im Sinne der modernen translationalen Medizin dringend benötigt (21).

Damit der Schritt von der Maus mit ihrer durchaus etwas anderen Biologie auf den Menschen nicht misslingt, wird sogar versucht, einen Zwischenschritt einzulegen und entsprechende erfolgreiche Ergebnisse

13 Als Präbiotika bezeichnet man in der Nahrung enthaltene Stoffe, die das Wachstum bzw. die Aktivität von Mikroorganismen (Bakterien oder Pilze) im Darm günstig beeinflussen. Ihr Status als Nahrungsergänzungsstoffe ist irgendwo zwischen Nahrungs- und Arzneimitteln angesiedelt. Die bringt angesichts der vielen neuen Erkenntnisse zur Funktion des Microbioms im Darm Probleme bei ihrer gesetzlichen Regulierung mit sich, die z.B. im Fachblatt Science diskutiert werden (22). Hiermit nicht verwechseln sollte man Probiotika, d. h. die mit der Nahrung (z. B. Yoghurt oder Sauerkraut) aufgenommene Mikroorganismen, welche die Darmflora positiv beeinflussen sollen. Nachweise für die seit mehr als hundert Jahren postulierten positiven Effekte stehen bislang aus oder werden kontrovers diskutiert $(42,43)$. 
zu den Auswirkungen probiotisch wirksamer Nahrungsbestandteile zunächst beim Schwein (mit seinem dem Menschen viel ähnlicheren Metabolismus) zu replizieren, bevor man schwer kranke Kinder in großen Zahlen behandelt. „Bringen Sie mal die 10 oder 12 Frischlinge einer $300 \mathrm{~kg}$ schweren Muttersau keimfrei - also ohne einen Atemzug - zur Welt und dann sofort in eine keimfreie Laborumgebung “, so der sichtlich emotionale Gordon bei seiner Präsentation vor einigen Wochen, „dann können Sie sich ausmalen, dass das kein einfacher Job ist" (21).

Der von Gordon und Mitarbeitern eingeschlagene tierexperimentelle Weg mit Hilfe von zunächst keimfrei aufgezogenen Tieren ist beim gegenwärtigen Forschungsstand nicht nur unausweichlich, sondern auch wesentlich eleganter als die Fäkaltransplantate (fecal transplants) von Mensch zu Mensch (z. B. mittels Einlauf), die man seit Jahren insbesondere in den USA durchführt. Dort wird nicht Unterernährung behandelt, sondern nicht zuletzt deren Gegenteil: Übergewicht $(20,35)$.

Weil in jüngerer Zeit eine rasch wachsende Zahl von Krankheiten mit einer Dysbiose des Darms - so nennt man dessen pathologische bzw. pathogene Besiedelung - in Verbindung gebracht wird, wundert nicht, dass das Verfahren der Übertragung von Bakterien aus dem Darm eines gesunden Spenders in den Darm eines kranken Empfängers zwischenzeitlich weitgehend unreguliert bei vielen „aussichtslosen Fällen" eingesetzt wurde und wird. Mittlerweile liegen sogar Übersichtsarbeiten zur Selektion des richtigen Spenders (68), zu laufenden, größeren, kontrollierten klinischen Studien (35) sowie zu dessen Risiken und Nebenwirkungen vor (66).

Die Verwendung von gnotobiotischen Tiermodellen zum Studium der Zusammenhänge von Darmflora und Krankheit ist wissenschaftlich ergiebiger als die ungezügelte „Ekeltherapie”.

Die Verwendung von gnotobiotischen Tiermodellen zum Studium der Zusammenhänge von Darmflora und Krankheit ist jedoch wissenschaftlich ergiebiger als die ungezügelte „Ekeltherapie“ (2), wie Fä-

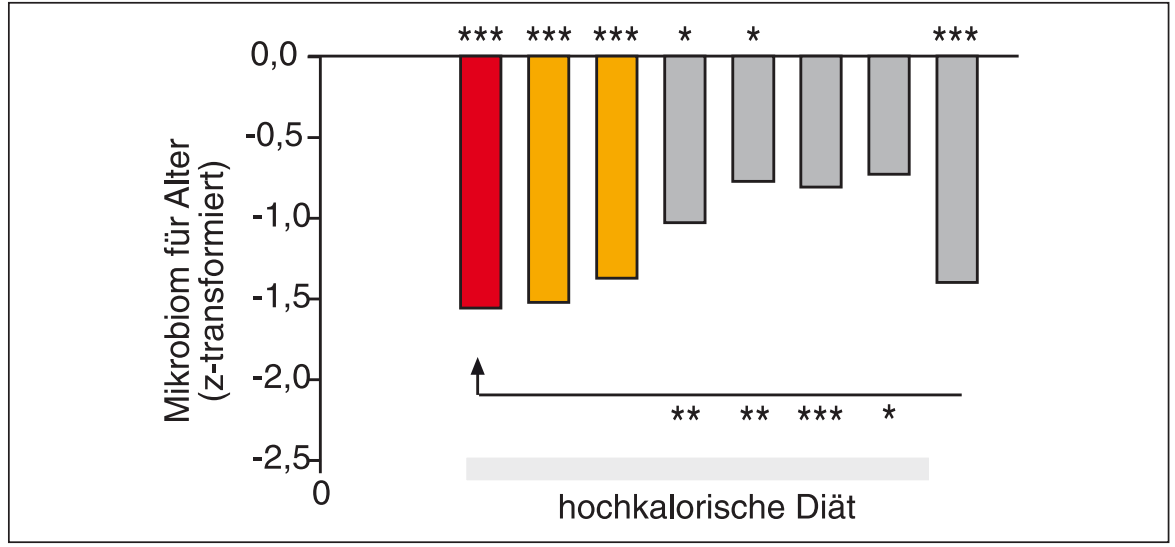

Abb. 3 Verminderung der Reifung des Microbioms von Kindern mit schwerer akuter Unterernährung (als Abweichung zum Reifungszustand des Microbioms gesunder Kinder aus derselben Region: Null-Linie) über den Verlauf der Untersuchung, von der Aufnahme über die therapeutische hochkalorische Diät (für im Mittel $10 \pm 5$ Tage) bis zu fünf Follow-up Untersuchungen in den Monaten danach (nach 72, S. 3, Figure 2b, links-unterer Quadrant); ${ }^{*} p<0,05 ;{ }^{* *} p<0,01$; ${ }^{* * *} p<0,001$; die $p$-Werte oben beziehen sich auf die Abweichung der jeweiligen Säule von der Null-Linie (Vergleich zu gesunden Kindern im Alter von über 6 Monaten) nach unten, die $p$-Werte unten beziehen sich auf den Vergleich der jeweiligen grauen Säulen mit der linken roten Säule.

kaltransplantate hierzulande gelegentlich nicht ganz emotionsfrei bezeichnet werden. Aber auch wenn die englischsprachigen Ärzte generell nur kühl-objektivierend von fecal transplant sprechen, lässt das zuweilen bei ihren Patienten - also selbst bei den pragmatischen Briten und Amerikanern - ein Unbehagen und die Assoziationen zu Schmutz, mangelnder Hygiene und sogar Ansteckung mit Krankheit aufkommen. Entsprechend gibt es Bestrebungen, anstatt von FMT (fecal microbial transplant) von GMT (gut microbial transplant) zu sprechen, um Vorurteile und zeitraubende Diskussionen mit besorgten Patienten und deren Angehörigen zu vermeiden $(24,40)$. Es steht zu erwarten, dass mit dem besseren Verständnis der kausalen Zusammenhänge bei Dysbiose die Notwendigkeit, FMT bzw. (semantisch eingängiger) GMT durchzuführen, abnimmt. Magensaftresistente Kapseln mit ganz bestimmten Bakterien befinden sich in der Erprobung.

Gnotobiotische Tiermodelle sind jedoch mehr als Werkzeuge für die Forschung. Sie stellen auch ein schönes Beispiel für personalisierte Medizin dar, lässt sich doch beispielsweise ein ausbleibender Therapieerfolg (z. B. in der Behandlung von Untergewicht in einem bestimmten Einzelfall) ganz einfach durch eine noch- malige Untersuchung der gleichen (zuvor eingefrorenen) Stuhlprobe des betroffenen Patienten am sterilen Mausmodell replizieren und experimentell auf unterschiedliche Weise beeinflussen (21). So ließen sich beispielsweise bei schweren therapierefraktären Durchfällen prä- oder probiotische Therapien zunächst am Modell erproben, um dann die geeignete Therapie auf diesen Einzelfall anzuwenden. Auch können die Auswirkungen von defizitären Diäten ( $\mathrm{z}$. B. Mangel von Vitamin A oder $B_{9}$, Eisen oder Zink) aus das menschliche Microbiom im Tierversuch studiert und diese Erkenntnisse im Rahmen einer translationalen Medizin auf den Menschen angewendet werden (23).

Aus evolutionärer Sicht ist zudem interessant, dass die Erkenntnissee zum Microbiom - neben der heterozygoten Fitness oder dem Zusammenspiel von Parasiten und Wirten - ein weiteres Beispiel für das komplexe Zusammenwirken von Organismus, Umwelt und Krankheit darstellen. Die heterozygote Fitness erklärt, warum es Sichelzellanämie dort gibt, wo es auch Malaria gibt und die Parasitologie erklärt, warum Toxoplasmose-positive Menschen aggressiver sind und eine geringere Abneigung gegenüber Katzenurin haben (55). Übrigens haben Malaria-positive Anopheles-Mücken auch eine besondere Vorliebe 
für Menschen, wie kürzlich nachgewiesen wurde (3). ${ }^{14}$

Ob ein bestimmtes Microbiom für die Gesundheit "gut" oder „schlecht" ist, hängt von den jeweiligen Umständen ab.

Die Auswirkungen des Darm-Microbioms auf unsere Gesundheit sind in ganz ähnlicher Weise komplex: Werden beispielsweise Nährstoffe durch Darmbakterien nicht aufgeschlossen, resultiert Mangelernährung in Gegenden, in denen die Nahrung ohnehin knapp ist. In Gegenden mit Nahrungsüberfluss hingegen kann dieser „Mangel“ an Enzymen des Microbioms vor Krankheit schützen, wie neueste Studien zur nicht Alkohol-bedingten Leberverfettung (non-alcoholic fatty liver disease, NAFLD) nahelegen (47). Je weniger für den Stoffwechsel des Menschen unverdauliche Zellulosefasern durch das Microbiom in (energiereiche) kurzkettige Fettsäuren umgewandelt werden, desto weniger Energie (in Form von Fett) muss die Leber speichern, was mit einem verminderten Risiko für ihre Verfettung (NAFLD) einhergeht. $\mathrm{Ob}$ ein bestimmtes Microbiom also für die Gesundheit „gut“ oder „schlecht“ ist, hängt von den jeweiligen Umständen ab.

Im Hinblick auf das Beispiel der NAFLD kommt hinzu, dass eine Dysbiose des Darms mit einer erhöhten Freisetzung von Endotoxinen und zugleich einer erhöhten Durchlässigkeit der Darmwand für diese Endotoxine einhergeht. Damit hat die erhöhte Belastung der Leber mit Toxinen über das Pfortaderblut gleich zwei Ursachen! Darüber hinaus kann Dysbiose auch bedeuten, dass mehr Bakterien im Darm vorhanden sind, die Äthanol produzieren. Damit stellt sich sogar die Frage, ob nicht die NAFLD (die ja definitionsgemäß nicht durch alimentär aufgenommenen Alkohol bedingt ist) letztlich doch durch (von den

14 „[...] the human malaria parasite P. falciparum evolved the ability to enhance transmission toward human, the appropriate host species, by increasing mosquito anthropophagy (or decreasing zoophagy) with potentially profound public health consequences" schreiben die Autoren am Ende ihrer sehr gründlichen Originalarbeit vom 21. Oktober 2017 (61).
Darmbakterien produzierten) Alkohol zumindest mitbedingt sein könnte (47). ${ }^{15}$

Halten wir fest: Die Wissenschaft zum lebendigen Inhalt unseres Dickdarms bringt derzeit fast wöchentlich neue, unerwartete und vor allem äußerst praxisrelevante Erkenntnisse hervor, von denen wir noch vor wenigen Jahren nicht einmal geträumt hätten. Veränderungen des DarmMicrobioms wurden mit einer ganzen Reihe weiterer Krankheiten in Verbindung gebracht. Hierzu zählen neben Mangelernährung auch Übergewicht und metabolisches Syndrom, entzündliche Darmerkrankungen und Darmtumoren einschließlich Darmkrebs; auch chronische Gelenkentzündungen (Arthritis) werden mittlerweile mit einer Dysbiose des Darms in Verbindung gebracht.

\section{Veränderungen des Darm-Micro- bioms wurden mit einer ganzen Reihe weiterer Krankheiten in Verbindung gebracht.}

Dass sogar die Gehirnentwicklung (60) und mithin bestimmte Krankheiten aus dem Bereich der Nervenheilkunde (ALS, Autismus, Depression, Stress, Morbus Alzheimer; 48) zu Veränderungen des Microbioms in Beziehung gebracht wurden, wundert im Grunde nicht, bestimmen doch unsere Mitbewohner eine Unzahl von Stoffwechselwegen mit salutogenen und pathogenen Konsequenzen. In einem Mausmodell der ALS wurde mit 2\% Buttersäure (das Produkt von einigen Darmbakterien) versetztes Wasser therapeutisch gegen Dysbiose des Darms eingesetzt und hatte einen lebensverlängernden Effekt (71). In einem Mausmodell von chronischem sozialen Stress führte die orale Verabreichung von Lactobacillus rhamnosus $\mathrm{zu}$ einer Verminderung der Stresssymptome, ohne jedoch das Microbiom positiv zu verändern (9).

Man spricht mittlerweile auch schon von der microbiome-gut-brain (MGB)

15 Entsprechend kann man nicht nur Mangelernährung durch Übertragung von Bakterien aus dem Darm mangelernährter Menschen bei Mäusen produzieren, sondern auch Leberverfettung (NAFLD) bei Mäusen durch Übertragung von Bakterien aus dem Darm von Menschen mit NAFLD. axis, also der Microbiom-Darm-GehirnAchse (18, 30, 46). Die Mehrheit der entsprechenden Publikationen stammt aus den Jahren 2016 und 2017, sodass man noch etwas abwarten sollte, bis sich ein möglicher Hype von handfesten, durch Daten gestützten Erkenntnissen mit einigermaßen akzeptabler Deutlichkeit abgrenzen lässt. Wenn das der Fall ist, wird man mehr darüber in der Nervenheilkunde erfahren. Bis dahin sollte jeder noch zur Kenntnis nehmen, dass Sport beim Menschen zu einer Verbesserung des Microbioms führt (38) und fettreiche Nahrung ihm schadet.

\section{Literatur}

1. Ahmed T, Ali M, Ullah MM, Choudhury IA, Haque ME, Salam MA, Rabbani GH, Suskind RM, Fuchs GJ. Mortality in severely malnourished children with diarrhoea and use of a standardised management protocol. Lancet 1999; 353: 1919-1922.

2. Anders SA. Wer eine kaputte Darmflora mit dem Kot anderer Menschen kurieren will, geht ein Risiko ein Die Ekeltherapie. Berliner Zeitung, 3.5.2011 https://www.berliner-zeitung.de/15052044.

3. Anonymus. Parasite makes mosquitoes hunt out humans. New Scientist 2017; 236 (3151): 20.

4. Ansaloni L, Catena F, Pinna AD. What is the function of the human vermiform appendix?. Evolution-based surgery: a new perspective in the Darwinian year 2009. Eur Surg Res 2009; 43: 67-71.

5. Bakker GJ, Nieuwdorp M. Fecal Microbiota Transplantation: Therapeutic Potential for a Multitude of Diseases beyond Clostridium difficile. Microbiol Spectr 2017; 5(4); doi: 10.1128/microbiolspec.BAD-0008-2017.

6. Barratt MJ, Lebrilla C, Shapiro HY, Gordon JI. The gut microbiota, food science, and human nutrition: A timely marriage. Cell Host Microbe 2017; 22: 134-141.

7. Bashan A, Gibson TE, Friedman J, Carey VJ, Weiss ST, Hohmann EL, Liu YY. Universality of human microbial dynamics. Nature 2016; 534: 259-262.

8. Bergman EN. Energy contributions of volatile fatty acids from the gastrointestinal tract in various species. Physiol Rev 1990; 70: 567-590.

9. Bharwani A, Mian MF, Surette MG, Bienenstock J, Forsythe P. Oral treatment with Lactobacillus rhamnosus attenuates behavioural deficits and immune changes in chronic social stress. BMC Med 2017; $15: 7$

10. Bjørkhaug ST, Skar V, Medhus AW, Tollisen A, Bramness JG, Valeur J. Chronic alcohol overconsumption may alter gut microbial metabolism: a retrospective study of 719 13C-D-xylose breath test results. Microbial Ecology in Health \& Disease 2017; 28: 1301725.

11. Bouter KE, van Raalte DH, Groen AK, Nieuwdorp M. Role of the gut microbiome in the pathogenesis of obesity and obesity-related metabolic dysfunction. Gastroenterology 2017; 152: 1671-1678. 
12. Chambers ES, Viardot A, Psichas A, et al. Effects of targeted delivery of propionate to the human colon on appetite regulation, body weight maintenance and adiposity in overweight adults. Gut 2015; 64 : 1744-1754.

13. Collins FS et al. A vision for the future of genomics research: A blueprint for the genomic era. Nature 2003; 422: 1-13.

14. David LA, Maurice CF, Carmody RN, Gootenberg DB, Button JE, Wolfe BE, Ling AV, Devlin AS, Varma Y, Fischbach MA, Biddinger SB, Dutton RJ, Turnbaugh PJ. Diet rapidly and reproducibly alters the human gut microbiome. Nature 2014; 505: 559-563.

15. Donaldson GP, Lee SM, Mazmanian SK. Gut biogeography of the bacterial microbiota. Nat Rev Microbiol 2016; 14: 20-32.

16. Erny D, de Angelis ALH, Jaitin D, Wieghofer P, Staszewski O, David E, et al. Host microbiota constantly control maturation and function of microglia in the CNS. Nat Neurosci 2015; 8: 965-977.

17. Flint HJ. Gut microbial metabolites in health and disease. Gut Microbes 2016; 7: 187-188.

18. Fung TC, Olson CA, Hsiao EY. Interactions between the microbiota, immune and nervous systems in health and disease. Nat Neurosci 2017; 20: 145-155.

19. Gannett L. The Human Genome Project. The Stanford Encyclopedia of Philosophy. Edward N Zalta (eds) 2016 (https://plato.stanford.edu/ archives/sum2016/entries/human-genome/)

20. Gérard P. Gut microbiome and obesity. How to prove causality? Ann Am Thorac Soc 2017; 14 (Supplement_5): S354-S356.

21. Gordon JI. The gut microbiota and childhood undernutrition: Looking at human development from a microbiological perspective. Presidential Lecture gehalten am Montag, den 13.11.2017 von 17.15 bis $18.30 \mathrm{Uhr}$, auf dem Jahreskongress der Society for Neuroscience in Washington, DC.

22. Green JM, Barratt MJ, Kinch M, Gordon JI. Food and microbiota in the FDA regulatory framework. Science 2017; 357: 39-40.

23. Hibberd MC, Wu M, Rodionov DA, Li X, Cheng J, Griffin NW, Barratt MJ, Giannone RJ, Hettich RL, Osterman AL, Gordon JI. The effects of micronutrient deficiencies on bacterial species from the human gut microbiota. Sci Transl Med 2017; 9(390) pii: eaal4069.

24. Jayasinghe TN, Chiavaroli V, Holland DJ, Cutfield WS, O'Sullivan JM. The new era of treatment for obesity and metabolic disorders: Evidence and expectations for gut microbiome Transplantation. Front Cell Infect Microbiol 2016; 6: 15.

25. Jones RM. The influence of the gut microbiota on host physiology: In pursuit of mechanisms. Yale J Biol Med 2016; 89: 285-297.

26. Kooij IA, Sahami S, Meijer SL, Buskens CJ, Te Velde AA. The immunology of the vermiform appendix: a review of the literature. Clin Exp Immunol 2016; 186: 1-9.

27. Le Roy T, Llopis M, Lepage P, Bruneau A, Rabot S, Bevilacqua C, Martin P, Philippe C, Walker F, Bado A, Perlemuter G, Cassard-Doulcier AM, Gérard P. Intestinal microbiota determines development of non-alcoholic fatty liver disease in mice. Gut 2013; 62: 1787-1794

28. Ley RE, Peterson DA, Gordon JI. Ecological and evolutionary forces shaping microbial diversity in the Human Intestine. Cell 2006; 124: 837-848.
29. Ley RE, Turnbaugh PJ, Klein S, Gordon JI. Microbial ecology: human gut microbes associated with obesity. Nature 2006; 444: 1022-1023.

30. Lima-Ojeda JM, Rupprecht R, Baghai TC. „I Am I and My Bacterial Circumstances": Linking gut microbiome, neurodevelopment, and depression. Front Psychiatry 2017; 8: 153.

31. Lin CA, Boslaugh S, Ciliberto HM, Maleta K, Ashorn P, Briend A, Manary MJ. A prospective assessment of food and nutrient intake in a population of Malawian children at risk for kwashiorkor. J Pediatr Gastroenterol Nutr 2007; 44: 487-493.

32. Lin L, Zhang J. Role of intestinal microbiota and metabolites on gut homeostasis and human diseases. BMC Immunol 2017; 18: 2.

33. Lloyd-Price J, Mahurkar A, Rahnavard G, Crabtree J, Orvis J, Hall AB, Brady A, Creasy HH, McCracken C, Giglio MG, McDonald D, Franzosa EA, Knight R, White O, Huttenhower C. Strains, functions and dynamics in the expanded Human Microbiome Project. Nature 2017; 550: 61-66.

34. Lübbert C, Salzberger B, Mössner J. Fäkaler Mikrobiomtransfer. Der Internist 2017; 58: 456-468.

35. Marotz CA, Zarrinpar A. Treating obesity and metabolic syndrome with fecal microbiota transplantation. Yale J Biol Med 2016; 89: 383-388.

36. McNeil NI. The contribution of the large intestine to energy supplies in man. Am J Clin Nutr 1984; 39: 338-342.

37. Minalyan A, Gabrielyan L, Scott D, Jacobs J, Pisegna JR. The gastric and intestinal microbiome: Role of proton pump inhibitors. Curr Gastroenterol Rep 2017; 19: 42.

38. Monda V, Villano I, Messina A, Valenzano A, Esposito T, Moscatelli F, Viggiano A, Cibelli G, Chieffi S, Monda M, Messina G. Exercise modifies the gut microbiota with positive health effects Oxid Med Cell Longev 2017: 3831972; doi: 10.1155/2017/3831972.

39. Moreno-Indias I, Sánchez-Alcoholado L, GarcíaFuentes E, Cardona F, Queipo-Ortuño MI, Tinahones FJ. Insulin resistance is associated with specific gut microbiota in appendix samples from morbidly obese patients. Am J Transl Res 2016; 8 : 5672-5684.

40. Park L, Tzimas D, Price J, Mone A, Hirsh J, Poles M, Malter L, Chen LA. Perceptions of fecal microbiota transplantation: factors that predict acceptance. In: JW Freston Conference. 2016 http://www.gastro.org/pressroom/James_W._Fres ton_Abstract_Factors_that_Predict_Acceptance.pdf.

41. Psichas A, Sleeth ML, Murphy KG, Brooks L, Bewick GA, Hanyaloglu AC, Ghatei MA, Bloom SR, Frost G. The short chain fatty acid propionate stimulates GLP-1 and PYY secretion via free fatty acid receptor 2 in rodents. International Journal of Obesity 2015; 39: 424-429.

42. Rijkers GT, de Vos WM, Brummer R-J, Morelli L, Corthier G, Marteau P. Health benefits and health claims of probiotics: bridging science and marketing. British Journal of Nutrition 2011; 106: 1291-1296.

43. Salminen S, van Loveren H. Probiotics and prebiotics: health claim substantiation. Microbial Ecology in Health \& Disease 2012; 23: 18568.

44. Samuel BS, Gordon JI. A humanized gnotobiotic mouse model of host-archaeal bacterial mutualism. PNAS 2006; 103: 10011-10016.
45. Sanduzzi Zamparelli M, Compare D, Coccoli P, Rocco A, Nardone OM, Marrone G, Gasbarrini A, Grieco A, Nardone G, Miele L. The metabolic role of gut microbiota in the development of nonalcoholic fatty liver disease and cardiovascular disease. Int J Mol Sci 2016; 17 pii: E1225.

46. Schroeder BO, Bäckhed F. Signals from the gut microbiota to distant organs in physiology and disease. Nat Med 2016; 22: 1079-1089.

47. Scott A. Menace in the microbiota. Nature 2017; 551: S94-S95.

48. Selber-Hnatiw S, Rukundo B, Ahmadi M et al. Human gut microbiota: Toward an ecology of disease. Frontiers in Microbiology 2017; 8: 1265.

49. Sender R, Fuchs S, Milo R. Revised estimates for the number of human and bacteria cells in the body. PLoS Biol 2016; 14(8): e1002533.

50. Serino M, Fernández-Real JM, García-Fuentes E, Queipo-Ortuño M, Moreno-Navarrete JM, Sánchez A, Burcelin R, Tinahones F. The gut microbiota profile is associated with insulin action in humans. Acta Diabetol 2013; 50: 753-761.

51. Smith HF, Fisher RE, Everett ML, Thomas AD, Bollinger RR, Parker W. Comparative anatomy and phylogenetic distribution of the mammalian cecal appendix. J Evol Biol 2009; 22: 1984-1999.

52. Smith MI, Yatsunenko T, Manary MJ, Trehan I, Mkakosya R, Cheng J, Kau AL, Rich SS, Concannon P, Mychaleckyj JC, Liu J, Houpt E, Li JV, Holmes E, Nicholson J, Knights D, Ursell LK, Knight R, Gordon JI. Gut microbiomes of Malawian twin pairs discordant for kwashiorkor. Science 2013; 339: 548-554.

53. Spitzer M. Armut macht dumm. Nervenheilkunde 2016; 35: 252-261.

54. Spitzer M. Stunting: Gehirnentwicklung, Status und Statur. Zur Bedeutung der ersten tausend Tage im Leben eines Menschen. Nervenheilkunde 2016; 35: 516-518.

55. Spitzer M. Protozoen und Persönlichkeitsstörungen, die Psychiatrie und der Ursprung der Menschheit. Nervenheilkunde 2016; 35: 341-350.

56. Tasnim N, Abulizi N, Pither J, Hart MM, Gibson DL. Linking the gut microbial ecosystem with the environment: Does gut health depend on where we live? Front Microbiol 2017; 8: 1935.

57. The Human Microbiome Project Consortium. Structure, function and diversity of the healthy human microbiome. Nature 2012; 486: 207-214.

58. The Human Microbiome Project Consortium. A framework for human microbiome research. Nature 2012; 486: 215-221.

59. Thursby E, Juge N. Introduction to the human gut microbiota. Biochem J 2017; 474: 1823-1836.

60. Tognini P. Gut microbiota: A potential regulator of neurodevelopment. Front Cell Neurosci 2017; 11: 25.

61. Vantaux A, Yao F, Hien DF, Guissou E, Yameogo BK, Gouagna L-C, Fontenille D, Renaud F, Simard F, Costantini C, Thomas F, Mouline K, Roche B, Cohuet A, Dabiré KR, Lefèvre T. Field evidence for manipulation of mosquito host selection by the human malaria parasite, Plasmodium falciparum. bioRxiv first online, Oct. 21, 2017 doi: http://dx.doi.org/10.1101/207183.

62. Vassallo G, Mirijello A, Ferrulli A, Antonelli M, Landolfi R, Gasbarrini A, Addolorato G. Review article: Alcohol and gut microbiota - the possible role of gut microbiota modulation in the treatment 
of alcoholic liver disease. Aliment Pharmacol Ther 2015; 41: 917-927.

63. Venter JC et al. The sequence of the human genome. Science 2001; 291: 1304-1351.

64. Vogt NM, Kerby RL, Dill-McFarland KA, Harding SJ, Merluzzi AP, Johnson SC, Carlsson CM, Asthana S, Zetterberg H, Blennow K, Bendlin BB, Rey FE. Gut microbiome alterations in Alzheimer's disease. Sci Rep 2017; 7: 13537.

65. Wang H, Lee IS, Braun C, Enck P. Effect of probiotics on central nervous system functions in animals and humans: A Systematic Review. J Neurogastroenterol Motil 2016; 22: 589-605.

66. Wang S, Xu M, Wang W, Cao X, Piao M, Khan S, Yan F, Cao H, Wang B. Systematic Review: Adverse events of fecal microbiota transplantation. PLoS ONE 2016; 11(8): e0161174.
67. Williams CD, Oxon BM, Lond H. Kwashiorkor: A nutritional disease of children associated with a maize diet. The Lancet 1935; 226: 1151-1152.

68. Woodworth MH, Neish EM, Miller NS, Dhere T, Burd EM, Carpentieri C, Sitchenko KL, Kraft CS. Laboratory testing of donors and stool samples for fecal microbiota transplantation for recurrent Clostridium difficile infection. J Clin Microbiol 2017; 55: 1002-1010.

69. Wu GD, Chen J, Hoffmann C, Bittinger K, Chen YY, Keilbaugh SA, Bewtra M, Knights D, Walters WA, Knight R, Sinha R, Gilroy E, Gupta K, Baldassano R, Nessel L, Li H, Bushman FD, Lewis JD. Linking long-term dietary patterns with gut microbial enterotypes. Science 2011; 334: 105-108.

70. Yatsunenko T, Rey FE, Manary MJ, Trehan I, Dominguez-Bello MG, Contreras M, Magris M,
Hidalgo G, Baldassano RN, Anokhin AP, Heath AC, Warner B, Reeder J, Kuczynski J, Caporaso JG, Lozupone CA, Lauber C, Clemente JC, Knights D, Knight R, Gordon JI. Human gut microbiome viewed across age and geography. Nature 2012; 486: 222-227.

71. Zhang YG, Wu S, Yi J, Xia Y, Jin D, Zhou J, Sun J. Target intestinal microbiota to alleviate disease progression in amyotrophic lateral sclerosis. Clin Ther 2017; 39: 322-336.

72. Subramanian $S$ et al. Persistent gut microbiota immaturity in malnourished Bangladeshi children. Nature 2014; 510: 417-421.

73. Qin J et al. A human gut microbial gene catalogue established by metagenomic sequencing. Nature 2010; 464: 59-65.

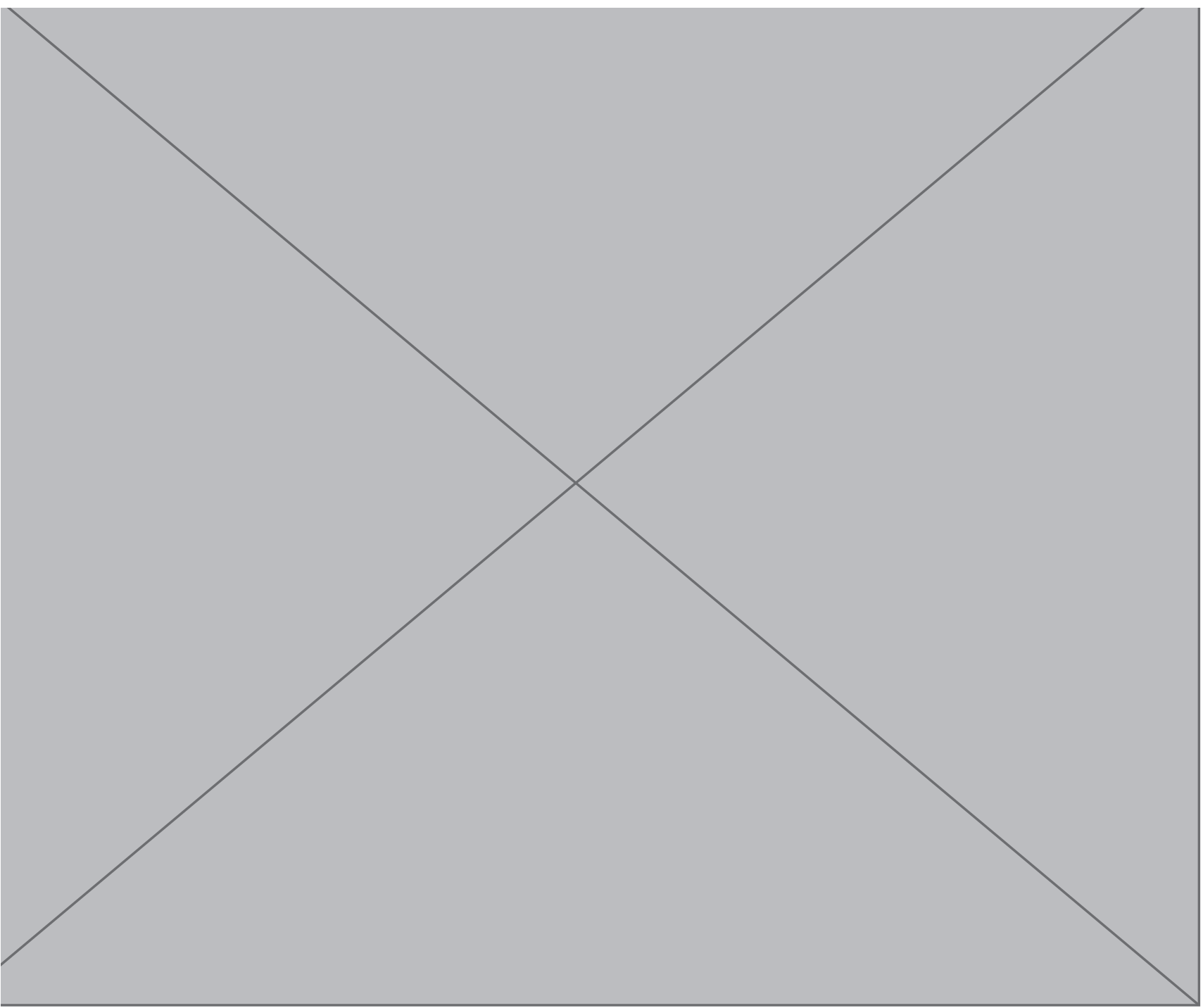

